\title{
VeA of Aspergillus niger increases spore dispersing capacity by impacting conidiophore architecture
}

\author{
Fengfeng Wang • Jan Dijksterhuis • \\ Timon Wyatt · Han A. B. Wösten • \\ Robert-Jan Bleichrodt
}

Received: 19 September 2014 / Accepted: 23 October 2014/Published online: 4 November 2014

(C) Springer International Publishing Switzerland 2014

\begin{abstract}
Aspergillus species are highly abundant fungi worldwide. Their conidia are among the most dominant fungal spores in the air. Conidia are formed in chains on the vesicle of the asexual reproductive structure called the conidiophore. Here, it is shown that the velvet protein VeA of Aspergillus niger maximizes the diameter of the vesicle and the spore chain length. The length and width of the conidiophore stalk and vesicle were reduced nearly twofold in a $\Delta v e A$ strain. The latter implies a fourfold reduced surface area to develop chains of spores. Over and above this, the
\end{abstract}

Electronic supplementary material The online version of this article (doi:10.1007/s10482-014-0316-z) contains supplementary material, which is available to authorized users.

F. Wang · H. A. B. Wösten $(\square)$ · R.-J. Bleichrodt Microbiology and Kluyver Centre for Genomics of Industrial Fermentation, Utrecht University, Padualaan 8, 3584, CH, Utrecht, The Netherlands

e-mail: h.a.b.wosten@uu.nl

F. Wang

e-mail: f.wang@uu.nl

R.-J. Bleichrodt

e-mail: rbleichrodt@gmail.com

J. Dijksterhuis - T. Wyatt

Applied and Industrial Mycology, CBS Fungal

Biodiversity Centre, Uppsalalaan 8, 3584, CT, Utrecht,

The Netherlands

e-mail: j.dijksterhuis@cbs.knaw.nl

T. Wyatt

e-mail: timonwyatt@gmail.com conidial chain length was approximately fivefold reduced. The calculated 20 -fold reduction in formation of conidia by $\Delta v e A$ fits the 8 - to 17 -fold decrease in counted spore numbers. Notably, morphology of the $\Delta v e A$ conidiophores of $A$. niger was very similar to that of wild-type Aspergillus sydowii. This suggests that $\mathrm{VeA}$ is key in conidiophore architecture diversity in the fungal kingdom. The finding that biomass formation of the A. niger $\Delta v e A$ strain was reduced twofold shows that $\mathrm{VeA}$ not only impacts dispersion capacity but also colonization capacity of A. niger.

Keywords Fungus - Aspergillus $\cdot$ VeA $\cdot$ Velvet protein $\cdot$ Asexual development $\cdot$ Conidiophore

\section{Introduction}

Aspergillus species are among the most abundant fungi in the world. They can grow in a wide range of abiotic growth conditions and can feed on a large variety of organic substrates (Krijgsheld et al. 2013). They grow predominantly saprobically but can also be pathogens of plants, animals and human. Colony formation starts with the germination of a sexual or asexual spore. Sexual reproduction is known in about one-third of the Aspergillus species including Aspergillus nidulans but excluding Aspergillus niger (Geiser 2009). In contrast, all aspergilli reproduce asexually by forming conidia. The high number and 
effective dispersal of these spores also explain the success of these fungi. Their conidia are among the most dominant fungal structures in the air (Bennett 2010).

Asexual development in A. nidulans mainly takes place in the light, whereas sexual development dominates in the dark (Purschwitz et al. 2006; Bayram et al. 2010). VeA plays an important role in this process. It is part of the velvet family that is highly conserved in ascomycetes and basidiomycetes (Bayram and Braus 2011). A $\Delta v e A$ strain of A. nidulans does not produce sexual fruiting bodies (i.e. cleistothecia) (Kim et al. 2002) but produces conidia both in the light and in the dark (Sarikaya Bayram et al. 2010). On the other hand, over-expression of veA reduces asexual development and increases production of cleistothecia. Thus, VeA is a positive regulator of sexual development and a suppressor of asexual development (Kim et al. 2002). VeA contains the typical velvet domain, a nuclear localization signal (NLS), a nuclear export signal (NES), and a protein degradation PEST (proline, glutamic acid, serine, and threonine) domain (Kim et al. 2002; Stinnett et al. 2007; Bayram and Braus 2011). VeA is predominantly localized in the cytoplasm in white or blue light, while it migrates to the nucleus in the dark or upon exposure to red light. Migration of VeA to the nucleus depends on the interaction between its NLS and the $\alpha$-importin KapA (Stinnett et al. 2007). Apart from KapA, VeA interacts with the regulator of secondary metabolism LaeA (Bok and Keller 2004) and the VeA-like protein VelB (Bok and Keller 2004; Bayram et al. 2008a). The $\mathrm{VeA} / \mathrm{VelB} / \mathrm{LaeA}$ complex mediates light regulated sexual and asexual development and links these processes to secondary metabolism (Bayram et al. 2008a).

The role of VeA varies in ascomycetes. For instance, VeA of $A$. parasiticus stimulates vegetative growth and production of conidia and sclerotia, and is involved in formation of the secondary metabolite aflatoxin (Calvo et al. 2004). Its homologue in $A$. fumigatus also stimulates asexual reproduction (Krappmann et al. 2005), while it affects microconidia macroconidia ratio, cell wall formation, and mycotoxin production in Fusarium verticilloides ( $\mathrm{Li}$ et al. 2006; Myung et al. 2009). Here, the role of VeA of $A$. niger was assessed. It is shown that this protein represses germination of conidia, promotes vegetative growth, and is involved in spatial position and morphology of conidiophores. As a result, the $\Delta v e A$ strain produces up to 17 times less spores. The conidiophore architecture of the $\Delta v e A$ strain suggests that the VeA regulatory pathway is key in diversity of conidiophore morphology in the fungal kingdom.

\section{Materials and methods}

Strains and growth conditions

A. niger strains used in this study (Supplemental Table 1) were grown at $30{ }^{\circ} \mathrm{C}$ in the dark or under constant white light (Osram Lumilux L36w/840, Osram, Munich, Germany) of 1200 lux. A. niger was grown on minimal medium (MM; $6 \mathrm{~g} \mathrm{l}^{-1} \mathrm{NaNO} 3,1.5 \mathrm{~g} \mathrm{l}^{-1}$ $\mathrm{KH}_{2} \mathrm{PO}_{4}, \quad 0.5 \mathrm{~g} \mathrm{l}^{-1} \mathrm{KCL}, 0.5 \mathrm{~g} \mathrm{l}^{-1} \quad \mathrm{MgSO}_{4} \cdot 7 \mathrm{H}_{2} \mathrm{O}$, $200 \mu \mathrm{l}$ trace element solution $\mathrm{l}^{-1}$ (Vishniac and Santer 1957), pH 6) with $1.5 \%$ agar and $25 \mathrm{mM}$ xylose. Colonies were grown as sandwiched cultures in a water saturated box (Wösten et al. 1991). To this end, the fungus was grown in between two perforated polycarbonate (PC) membranes (diameter of $76 \mathrm{~mm}$; pore size $0.1 \mu \mathrm{m}$; Osmonics, GE Water Technologies, Trevose, $\mathrm{PA})$ in a $0.2 \mathrm{~mm}$ thin layer of $1.25 \%$ agarose. Sandwiched cultures were inoculated in the centre of the PC membrane with $2 \mu \mathrm{l}$ of spore suspension $(1,000$ spores in total in saline Tween [0.8\% NaCl, $0.005 \%$ Tween-80]). The top membrane was positioned on the agarose layer after $24 \mathrm{~h}$ of incubation.

\section{Vectors}

\section{Construction of the veA deletion vector}

Up- and downstream flanking regions and the coding sequence of veA (ANI_1_738074) were amplified from chromosomal DNA of A. niger N402 using accuTaq polymerase (Sigma-Aldrich, St. Louis, USA) and primer pair 1 (Supplemental Table 2). The amplified product was introduced in pGEM $^{\circledR}$-T easy (Promega, Madison, USA). The XbaI fragment including the veA coding sequence was replaced in the resulting plasmid by the XbaI fragment of pXDRFP4 (Yang et al. 2004) that contains the pyrG selection cassette. This resulted in the veA deletion construct $\mathrm{pVeAKO}$ that contains 604 bp upstream and $509 \mathrm{bp}$ downstream flanking sequence of $v e A$. 
Construction of the veA complementation vector

The coding sequence of $v e A$ and 1,037 bp upstream and $573 \mathrm{bp}$ downstream flanking sequences were amplified from chromosomal DNA of A. niger N402 using primer pair 2 (Supplemental Table 2) and Phusion ${ }^{\circledR}$ High-Fidelity DNA polymerase (BIOKÉ, Leiden, the Netherlands). As a result, a NotI and PciI site had been introduced at the $5^{\prime}$ and $3^{\prime}$ ends of the amplified fragment, respectively. These sites were used to introduce the PCR product into pGPDGFP (Lagopodi et al. 2002) that had been cut with the same enzymes. This resulted in the veA complementation construct $\mathrm{pVC}$.

\section{VeA TagRFP-T fusion construct}

TagRFP-T was amplified by PCR using Phusion ${ }^{\circledR}$ High-Fidelity DNA polymerase, primer pair 3 (Supplemental Table 2), and plasmid TagRFP-158T-C1 (Merzlyak et al. 2007) as template. The amplified fragment was inserted in the SmaI site of pUC19, cut from the resulting plasmid pRB068 with NcoI and HindIII, and introduced in pGPDGFP (Lagopodi et al. 2002) that had been digested with the same enzymes. This resulted in vector pRB069. The promoter and ORF of veA were amplified by PCR using primer pair 4, A. niger $\mathrm{N} 402$ chromosomal DNA as template, and Phusion ${ }^{\circledR}$ High-Fidelity DNA polymerase. NotI and PciI sites had been introduced at the $5^{\prime}$ and $3^{\prime}$ ends of the amplified fragment, respectively. The fragment was cut with these enzymes and introduced in between the NotI and NcoI sites of vector PRB069 in front of TagRFP-T and the $\operatorname{trpC}$ terminator. The resulting vector pVeARFP contains veA under control of its own promoter fused at its C-terminus with TagRFP-T.

\section{Construction of the nuclear GFP expression plasmid}

The $m p d A$ promoter (ANI_1_802024) was amplified from chromosomal DNA of A. niger N402 using primer pair 5 (Supplemental Table 2) and Phusion ${ }^{\circledR}$ High-Fidelity DNA polymerase. The PCR product was digested with NotI and NcoI and ligated with the $4 \mathrm{~kb}$ NotI/NcoI fragment of pCB034 (de Bekker et al. 2011) harboring the H2B-GFP fusion sequence. This plasmid was named pRB009.
Transformation

Transformation of A. niger was performed as described (de Bekker et al. 2009). Protoplasts of $A$. niger MA70.15 were generated using $40 \mathrm{mg}$ lysing enzyme (L-1412, Sigma-Aldrich, Zwijndrecht, Netherlands), $2 \mathrm{mg}$ chitinase (C6137, Sigma-Aldrich, Zwijndrecht, Netherlands), and $2 \mathrm{mg} \beta$-glucuronidase (G0751, Sigma-Aldrich, Zwijndrecht, Netherlands) per gram wet weight of mycelium. Transformants of MA70.15 in which pVeAKO was introduced were selected on MMS agar (MM containing per liter $325 \mathrm{~g}$ sucrose and $12 \mathrm{~g}$ agar). Inactivation of $v e A$ was confirmed by PCR analysis with primer pairs 6 and 7 (Supplemental Table 2). Transformants of the $\Delta v e A$ strain resulting from a co-transformation of plasmid pVC and pAN7-1 harboring the hygromycin resistance cassette (Punt et al. 1987) were selected on MMS medium with $200 \mu \mathrm{g} \mathrm{ml}^{-1}$ hygromycine and $50 \mu \mathrm{g} \mathrm{ml}^{-1}$ caffeine. Complementation was confirmed by PCR analysis using primer pair 6 (Supplemental Table 2). A similar selection was used for transformants of the $\Delta v e A$ strain resulting from the cotransformation of the reporter construct pveARFP and pAN7-1. Introduction of the reporter construct was confirmed by PCR using primer pair 6 (Supplemental Table 2). Transformants expressing pVeARFP were re-transformed with pRB009 and pRB206 (Bleichrodt 2012) and screened on MMS medium with $100 \mu \mathrm{g} \mathrm{ml}^{-1}$ phleomycin. The former plasmid encompasses the histone 2B-GFP fusion, while the latter plasmid contains the phleomycin resistance cassette. To construct pRB206, the ORF of the phleomycin resistance gene was amplified from pGEMphleoB-hom2 (Ohm et al. 2010) using primer pair 8. It was cut with NcoI and HindIII and ligated in pGPDGFP (Lagopodi et al. 2002) that had been cut with the same enzymes. The resulting vector pRB206 contains the phleomycin resistance gene under control of the gpdA promoter of A. nidulans and the $\operatorname{trpC}$ terminator.

\section{Light microscopy}

Cultures were grown in glass bottom dishes in the light (MatTek, www.glass-bottom-dishes.com, P35G-1.520-C, Ashland, USA) under water saturated conditions. To this end, glass bottom dishes were filled with $30 \mu \mathrm{l}$ MM containing $1 \%$ agarose and $25 \mathrm{mM}$ xylose 
(pre-warmed at $60{ }^{\circ} \mathrm{C}$ ). A round cover slip (diameter $18 \mathrm{~mm}$ ) was placed on top of the medium and $0.5 \mu \mathrm{l}$ of spore suspension (500 spores $\mu \mathrm{l}^{-1}$ ) was placed next to the cover slip after the medium had solidified. The agar medium was overlaid with $2 \mathrm{ml}$ liquid $\mathrm{MM}$ with $25 \mathrm{mM}$ xylose. Hyphae had formed after 3 days in the agar medium. Germination, growth and development were monitored with a MULTIZOOM AZ100 microscope (Nikon Corporation Instruments, Tokyo, Japan).

\section{Fluorescence microscopy}

Pieces from 7-day-old sandwiched colonies $(10 \times 10 \mathrm{~mm})$ were excised and placed up-side-down on an object glass after the upper PC membrane was removed. RFP and GFP fluorescence were monitored on a Zeiss LSM 700 laser scanning confocal microscope (Carl Zeiss Micro-Imaging, Oberkochen, Germany). GFP was excited with the $488 \mathrm{~nm}$ laser line and fluorescence was detected at 490-555 nm band pass. RFP was excited with a $555 \mathrm{~nm}$ laser line and monitored using a $560 \mathrm{~nm}$ long pass. Images were captured as z-series of optical sections $(\sim 1 \mu \mathrm{m}$ for hyphae and $\sim 2 \mu \mathrm{m}$ for conidiophores). Data sets were displayed as maximum intensity projections $(1,024 \times 1,024$ pixels $)$. Images were processed using ZEN 2010 software.

RFP images were captured using a Plan-Apochromat $63 \mathrm{x} / 1.40$ Oil DIC M27 objective with a pixel time of $1.58 \mu \mathrm{s}$, the laser at $3 \mathrm{~mW}$, and a pinhole of $70 \mu \mathrm{m}$. In case of co-localization of GFP and RFP expressing strains grown in the light, images were captured with the same objective and pixel time but the $488 \mathrm{~nm}$ laser was set at $0.6 \mathrm{~mW}$ and the $555 \mathrm{~nm}$ laser at $1.7 \mathrm{~mW}$. The pinhole was set at 44 and $48 \mu \mathrm{m}$, respectively. In case of co-localization of GFP and RFP expressing strains grown in the dark, images were captured with a Plan-Neofluor 40x/1.30 Oil DIC objective. The pixel time was set at $0.79 \mu \mathrm{s}$, the $488 \mathrm{~nm}$ laser at $1.3 \mathrm{~mW}$, the $555 \mathrm{~nm}$ laser at $1 \mathrm{~mW}$, and the pinholes at 33 and $36 \mu \mathrm{m}$, respectively.

\section{Scanning electron microscopy}

Sandwiched colonies were grown for 7 days in the absence of an agarose layer. Sections of the colonies were cut by means of a stainless razor blade, and glued in a copper cup (1 cm diameter) with KP-Cryoblock
(Klinipath, Duiven, the Netherlands). Samples were viewed with a JEOL 5600LV scanning electron microscope (JEOL, Tokyo, Japan) via an Oxford CT1500 Cryostation as described (Wyatt et al. 2014).

Quantification of sporulation

The upper PC membrane was removed from 3- or 6-day-old sandwiched colonies grown in the dark or in the light. Growth in the dark or in the light was prolonged for $24 \mathrm{~h}$, after which spores were harvested and counted using a haemocytometer. The spore concentration was related to the surface area of the colony. Spore counting was done in duplo with colonies with same diameter (4-day-old wild-type colonies and 7-day-old $\Delta v e A$ colonies) and in quadruple with colonies with same age (7-day-old colonies).

Biomass analysis

Sandwiched colonies were grown in the light and in the dark in the absence of an agarose layer. Biomass of colonies was determined in triplicate after freezedrying. To obtain similar colony diameter, the upper PC membrane of wild-type colonies was removed after 3 days and that of $\Delta v e A$ colonies after 6 days of growth.

Sugar analysis

Colonies were grown as sandwiched cultures (see above). After 6 days of growth, the top membrane was either or not removed. Upon removal of the membrane, formation of aerial hyphae and conidiophores was induced. After $24 \mathrm{~h}$, vegetative mycelium (colonies grown between membranes), mycelium with conidiophores (colonies with upper membrane removed), and conidia were harvested. Samples were frozen in liquid nitrogen, freeze dried, and homogenized with the Qiagen Tissuelyser (2 min at 30 strokes s $^{-1}$ ) in a stainless steel grinding jar (Qiagen, Valencia, USA) cooled with liquid nitrogen. $1 \mathrm{ml}$ milli $\mathrm{Q}$ water was added and grinding was continued for an additional $2 \mathrm{~min}$ at 30 strokes s$^{-1}$. Samples were thawed, transferred to a $2 \mathrm{ml}$ Eppendorf tube and centrifuged at $4{ }^{\circ} \mathrm{C}$ for $30 \mathrm{~min}$ at $10,000 \mathrm{~g}$. The supernatant was heated for $30 \mathrm{~min}$ at $95^{\circ} \mathrm{C}$ and centrifuged for $30 \mathrm{~min}$ at $10,000 \mathrm{~g}$. The supernatant was filtered using a $0.2 \mu \mathrm{m}$ Acrodisc $\mathrm{Cr} 13 \mathrm{~mm}$ 


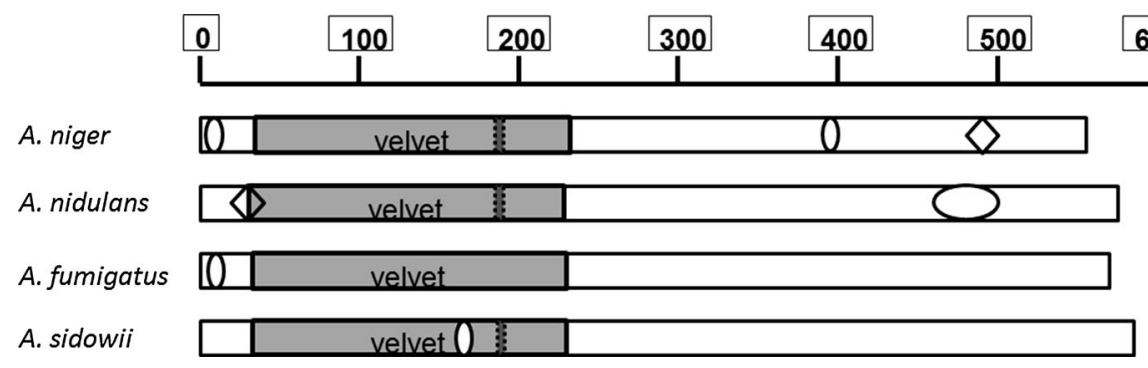

Fig. 1 Domains of VeA of A. niger, A. nidulans, A. fumigatus, and A. sydowii. NLS (diamant), NES (stippled box), and PEST (ovals) represent nuclear localization signal, nuclear export signal, and PEST sequences, respectively

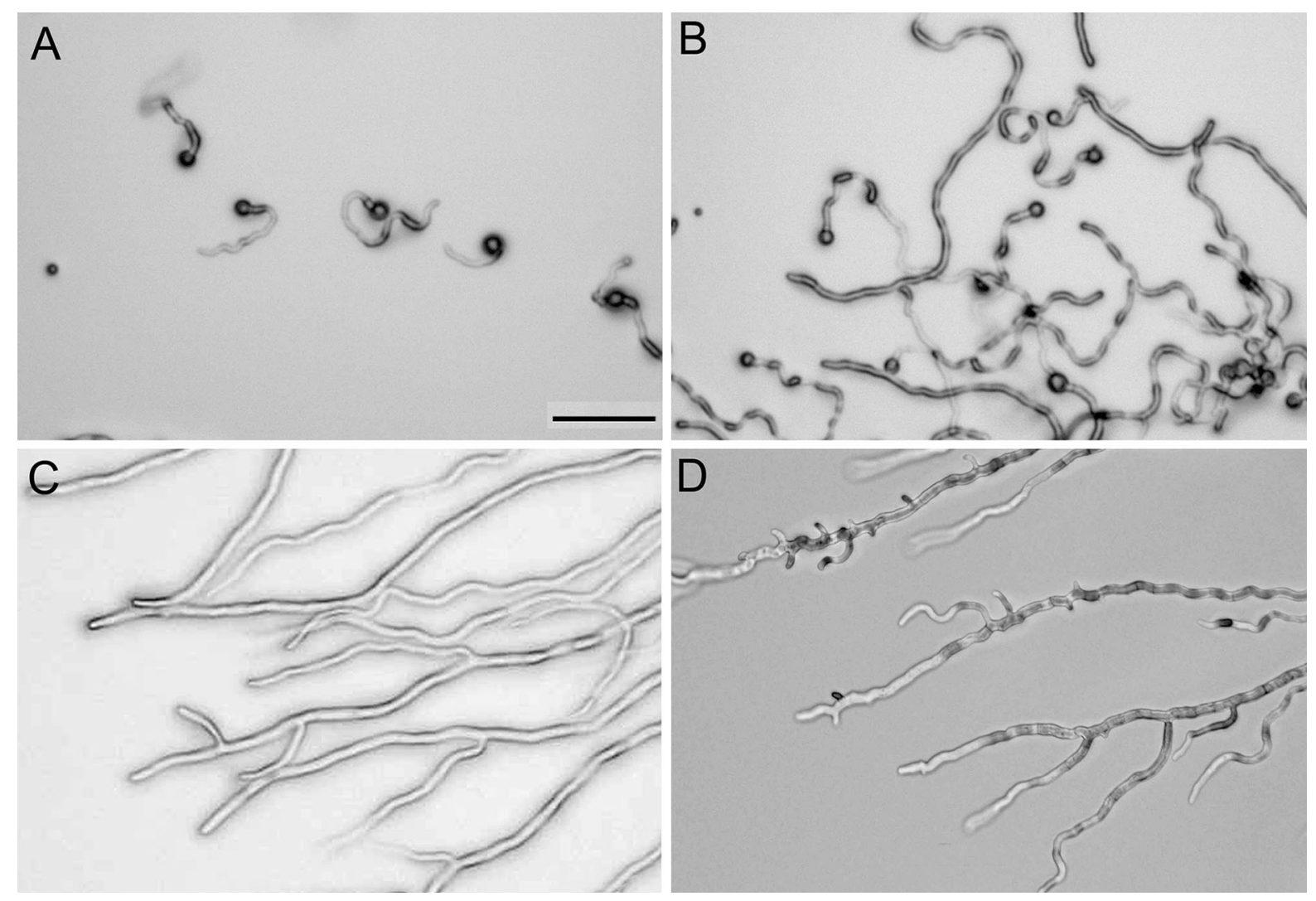

Fig. 2 Germination and hyphal growth of the control strain RB\#210.1 (a, c), and the $\Delta v e A$ strain (b, d) $16 \mathrm{~h}(\mathbf{a}, \mathbf{b})$ and $48 \mathrm{~h}(\mathbf{c}, \mathbf{d})$ after inoculation. Bar represents $50 \mu \mathrm{m}$

Syringe filter (Pall Life Science, Mijdrecht, The Netherlands). Quantitative analysis of saccharides and polyols was carried out in technical and biological triplicates by high-performance liquid chromatography (HPLC) equipped with a Sugar-Pak I cationexchange column (Waters, Etten-Leur, The
Netherlands). The column and detector were kept at $50{ }^{\circ} \mathrm{C}$ with a column heater (Waters) and internal heater, respectively. Samples were injected using the 717+ autosampler (Waters) and separated with

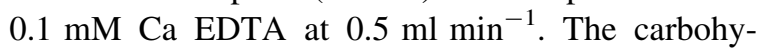
drates were detected with an IR detector $(2,414$ 

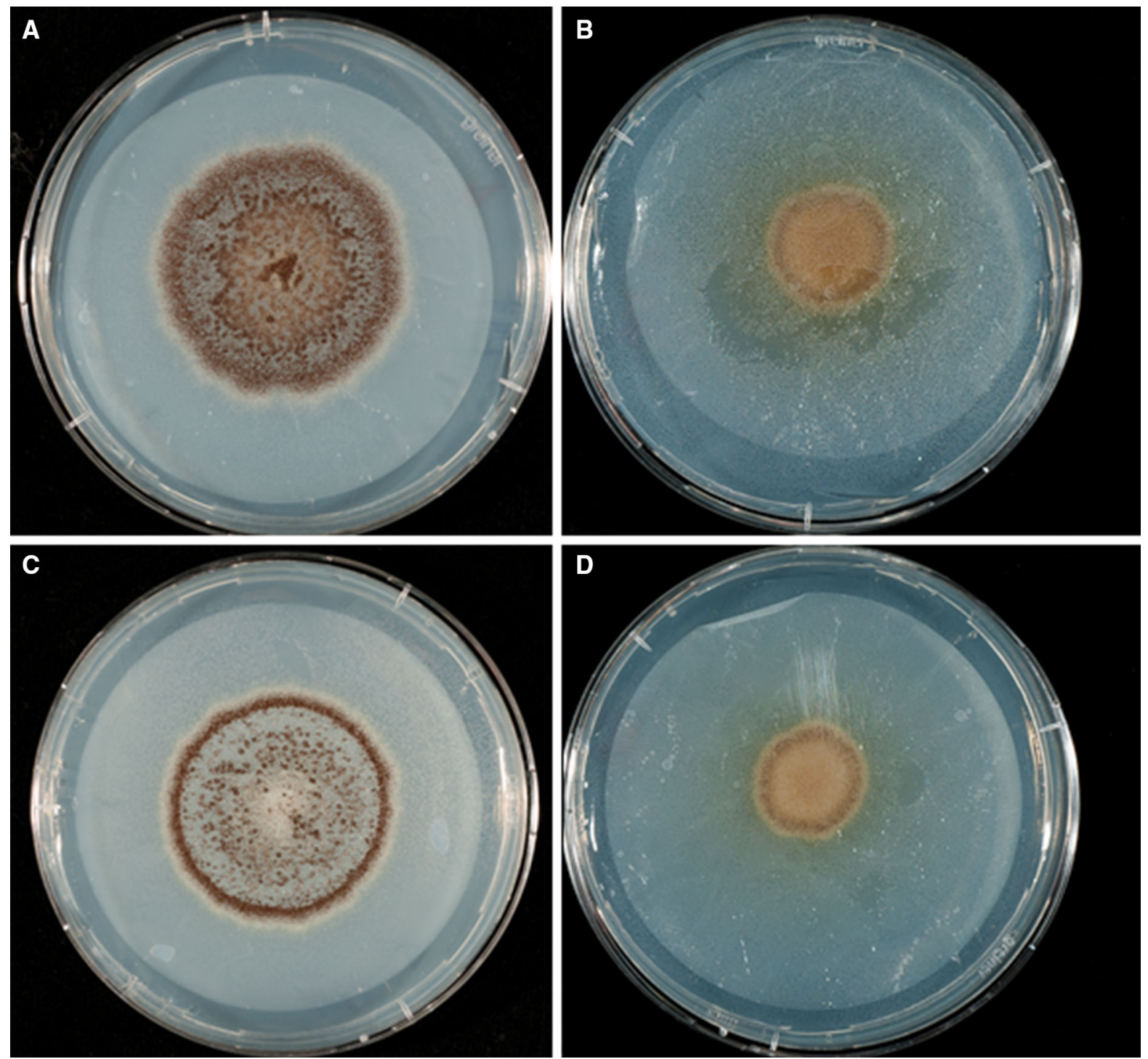

Fig. 3 Radial growth and sporulation profile of 7-day-old xylose grown sandwiched colonies of the control strain $\mathrm{RB} \# 210.1(\mathbf{a}, \mathbf{c})$, and the $\Delta v e A$ strain $(\mathbf{b}, \mathbf{d})$ from which the

refractive index detector, Waters). Peak integrations and calculation were performed with Empower software delivered by Waters. The retention time of the peaks were compared with those of $0.01-0.50 \% \mathrm{w} / \mathrm{v}$ trehalose, mannitol, glucose, glycerol, erythritol and arabitol.

Statistical analysis

Statistical analysis was performed with IBM Statistics 20 (SPSS statistics; IBM New York, USA).

upper PC membrane was removed at day 6. Colonies were grown continuously in the light $(\mathbf{a}, \mathbf{b})$ or in the dark $(\mathbf{c}, \mathbf{d})$

\section{Results}

Gene VeA of A. niger

Gene An08g05100 (ANI_1_738074) was annotated as the veA homologue of $A$. niger (Pel et al. 2007). It encodes a protein of 555 amino acids that shares $58 \%$ identity and $69 \%$ similarity with the 573 amino acid long VeA protein of $A$. nidulans. Amino acid identity in the velvet domain is even $72 \%$. Like VeA of A. nidulans, VeA of A. niger contains three short PEST 


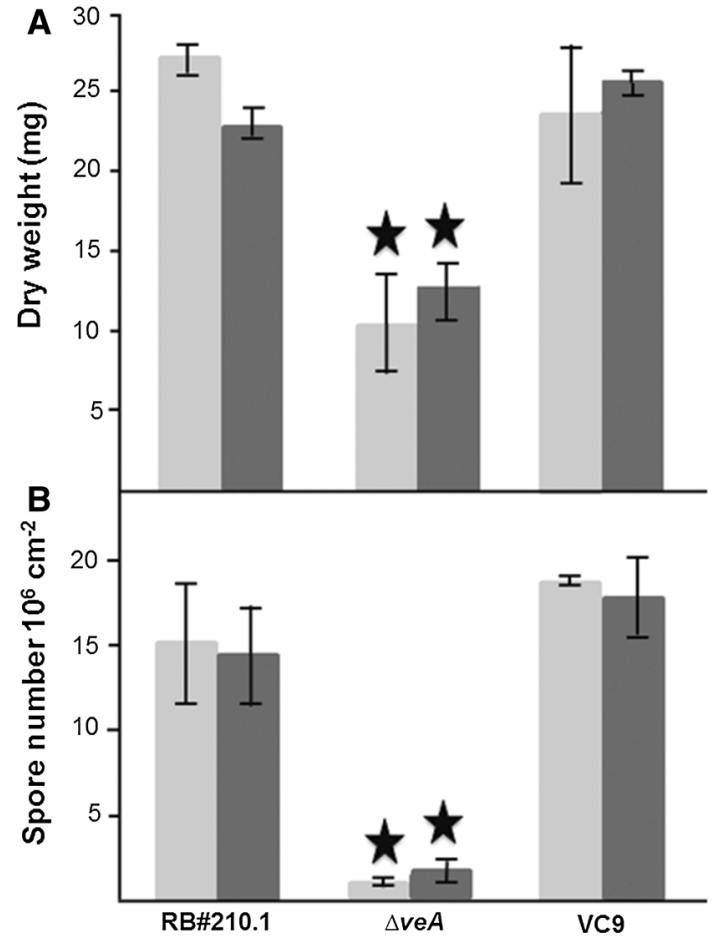

Fig. 4 Dry weight (a) and spore numbers (b) of sandwiched colonies of the control strain RB\#210.1, $\Delta v e A$, and the $\Delta v e A$ complemented strain VC9 that had been grown on xylose in the light (light gray shading) or in the dark (dark gray shading). Cultures in a had been grown for 7 days between polycarbonate membranes. Sandwiched colonies in $\mathbf{b}$ of the control and VC9 had been grown for 3 days on xylose medium, while those of $\Delta v e A$ had been grown for 6 days before the upper PC membrane was removed to allow conidia formation during a $24 \mathrm{~h}$ period. In this time interval all colonies had a diameter of about $20 \mathrm{~mm}$. Asterisk indicates significant difference to RB\#210.1 and VC9 (ANOVA $p<0.05$ )

sequences, a NES, and is predicted to have a nuclear localization according to PSORT II (Horton and Nakai 1997)(Fig. 1). However, a clear localization signal is absent.

\section{Inactivation of $\mathrm{VeA}$ of $A$. niger}

The $v e A$ deletion vector $\mathrm{pVeAKO}$ was introduced in $A$. niger strain MA70.15. Transformants were screened by PCR resulting in $4 \Delta v e A$ strains. The coding sequence of $v e A$ could not be amplified in these strains. In contrast, fragments were obtained with primer pairs annealing in the pyrG cassette and upstream of the $5^{\prime}$ flanking sequence and in the $p y r G$ cassette and downstream of the $3^{\prime}$ flanking sequence.
These fragments could not be amplified from the genomic DNA of the parental strain (Supplemental Fig. 1). All strains showed the same phenotype. Strain $\Delta v e A 6$ was selected for further analysis. This strain was complemented by introduction of $\mathrm{pVC}$ that contains an intact copy of veA.

\section{Functional analysis of VeA of A. niger}

Conidia of the $\Delta v e A$ strain germinated faster when compared to the control strain RB\#210.1 and the complemented $\triangle v e A$ strain VC9 when grown on xylose (data not shown) or in the absence of carbon source (Fig. 2). In contrast, the $\Delta v e A$ strain showed reduced radial growth on xylose when compared to the control strain RB\#210.1 (Fig. 3) and the complemented $\triangle v e A$ strain VC9 (data not shown). Reduced radial growth of the $\Delta v e A$ strain was accompanied by wavy-like growth of hyphae (Fig. 2). Moreover, the biomass of 7-day-old $\Delta v e A$ colonies was approximately twofold lower when compared to the wild-type and the complemented $\Delta v e A$ strain (Fig. 4).

Seven-day-old sandwiched colonies of the $\Delta v e A$ strain had mainly formed conidiophores in the subperipheral zone of the mycelium when the upper PC membrane had been removed at day 6 (Fig. 3). The conidiophore density in the center of $\Delta v e A$ colonies was much less when compared to the control and the complemented strain. Conidiophore formation was also assessed in 4-day-old wild-type colonies to exclude that the spatial difference in conidiophore formation in the $\Delta v e A$ strains is due to their reduced diameter. These control colonies with a similar diameter as 7-day-old $\Delta v e A$ colonies also showed the two sporulation zones in the center and the periphery of the colony (data not shown).

Conidiophore formation was affected in strain $\Delta v e A$ (Fig. 5) when compared to the wild type and the complemented VC9 strain (data not shown). The height of the stalks of $\Delta v e A$ was $123 \mu \mathrm{m}$, while that of VC9 and the control strain was 199 and $225 \mu \mathrm{m}$, respectively (Table 1 ). The width of the stalk and the vesicle was also reduced when compared to the control strain and VC9. The stalk width of $\Delta v e A$ was $6 \mu \mathrm{m}$, while that of the control and the complemented $\Delta v e A$ strain VC9 was 11 and $10 \mu \mathrm{m}$, respectively. Vesicle diameter of $\Delta v e A$ was twofold reduced when compared to the wild-type and the complemented strain, while diameter of the conidiophore of $\Delta v e A$ was even 

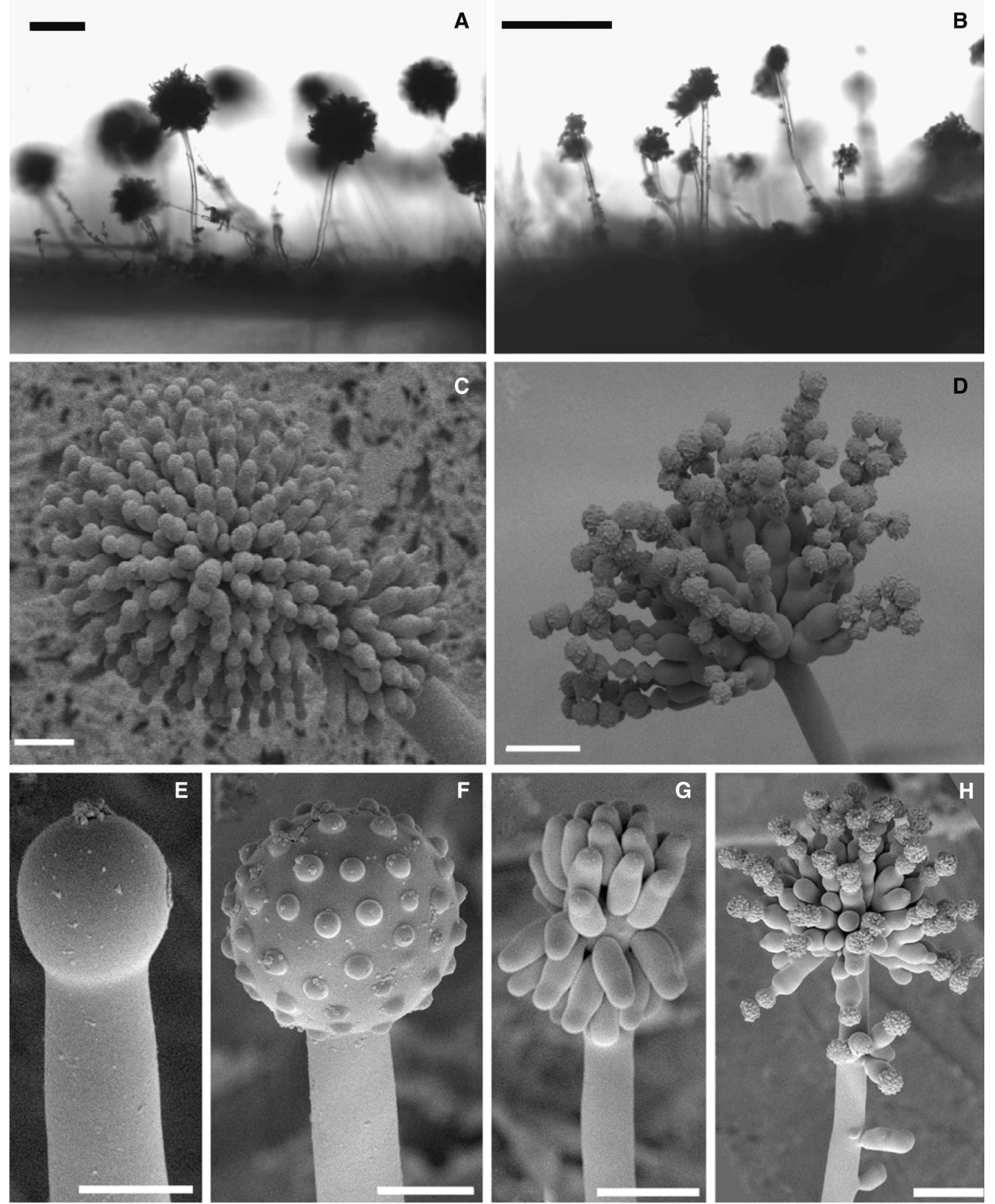

Fig. 5 Light (a, b) and scanning electron (c-h) microscopy of conidiophores of the control strain RB\#210.1 (a, c), and $\Delta v e A(\mathbf{b}, \mathbf{d}-\mathbf{h})$. Bar represents $100 \mu \mathrm{m}(\mathbf{a}, \mathbf{b})$, and $10 \mu \mathrm{m}(\mathbf{c}-\mathbf{h})$ 
Table 1 Length and width $(\mu \mathrm{m})$ of conidiophore stalks and diameter $(\mu \mathrm{m})$ of vesicles and conidiophore of the control strain $\mathrm{RB} \# 210.1, \Delta v e A$, and the complemented $\Delta v e A$ strain VC9. Averages are the result of quintuplicate experiments

\begin{tabular}{lccc}
\hline & RB\#210.1 & $\Delta v e A$ & VC9 \\
\hline Stalk length & $225 \pm 39$ & $123 \pm 39$ & $199 \pm 61$ \\
Stalk width & $11 \pm 2$ & $6 \pm 1$ & $10 \pm 1$ \\
Vesicle diameter & $17 \pm 5$ & $8 \pm 2$ & $16 \pm 4$ \\
Conidiophore diameter & $150 \pm 34$ & $56 \pm 13$ & $151 \pm 27$ \\
\hline
\end{tabular}

threefold reduced (56 $\mu \mathrm{m}$ versus 150 and $151 \mu \mathrm{m}$, respectively) (Table 1). This and scanning electron microscopy (Fig. 5) showed that spore chain length is reduced in $\triangle v e A$.

The $\Delta v e A$ strain had formed more than 8 (in the dark) and 17 (in the light) times less conidia per surface area, respectively, when compared to the wild type and the complemented VC9 strain (Fig. 4). This result was obtained when colonies were taken that had a similar size (i.e. differing in age) or that had a similar age (i.e. differing in colony diameter). Light and dark conditions had no effect on spore numbers both in the control strains and in $\Delta v e A$.

\section{VeA localization}

Construct pVeARFP that encompasses the gene encoding the VeA-TagRFP-T fusion was introduced in the $\Delta v e A$ strain. Transformants were obtained that showed a wild-type phenotype, confirming that the fusion protein can replace wild-type VeA. Fluorescence of VeA-TagRFP-T was observed in vegetative mycelium and conidiophores of light- (Fig. 6) and dark- (data not shown) grown cultures. The fusion protein resided mainly in nuclei in the dark, while it dominated in the cytosol when cultures were grown in the light (Fig. 6). The nuclear location of VeA-RFP was confirmed by introducing the gene encoding nuclear targeted H2B-GFP in the pveARFP strain. Fluorescence of RFP and GFP co-localized.

Inactivation of $\mathrm{VeA}$ influences compatible solute accumulation

The presence of compatible solutes in vegetative mycelium, mycelium with conidiophores and conidia, and conidia of 7-day-old colonies of the control strain and $\triangle v e A$ was evaluated by means of HPLC analysis.
No differences between the strains were observed for glucose, glycerol and arabitol. In contrast, both vegetative mycelium and conidia of $\Delta v e A$ contained less trehalose when compared to wild type and the complemented strain (Tables 2, 3). Trehalose concentration had decreased about 4- and more than 10-fold in mycelium and mycelium with conidia, respectively, when compared to wild type (Table 2). Trehalose was even completely absent in conidia of $\Delta v e A$ (Table 3 ). Erythritol levels were 2- to 3-fold higher in mycelium and mycelium with conidiophores of strain $\Delta v e A$ when compared to wild type (Table 2). No difference in concentration of this polyol was observed in conidia (Table 3). In contrast, mannitol levels had been reduced twofold in $\Delta v e A$ conidia, while no difference was found in mycelium and mycelium with conidia. Notably, the total concentration of compatible solutes didn't show significant differences between wild type, $\Delta v e A$, and VC9 in mycelium and conidia (Table 2).

\section{Discussion}

VeA is conserved in Aspergillus and other fungi such as Fusarium verticillioides and Neurospora crassa (Li et al. 2006; Bayram et al. 2008b). The function of this velvet protein varies between fungi. We here showed that $\mathrm{VeA}$ of $A$. niger represses germination, stimulates hyphal growth, and is involved in spatial positioning and morphology of conidiophores. The latter explains why a $\triangle v e A$ strain produces less spores. Reduced spore numbers have also been observed in $\Delta v e A$ strains of $A$. parasiticus and A. fumigatus. It is tempting to speculate that this protein also determines conidiophore positioning and morphology in these aspergilli. The conidiophores of the $\Delta v e A$ strain of $A$. niger were strikingly similar to $A$. sydowii. This suggests that VeA is an important morphogen that is key in conidiophore architecture diversity in the fungal kingdom.

The $v e A$ gene of $A$. niger encodes a velvet protein that contains three short PEST sequences, a nuclear export signal, and is predicted to have a nuclear localization. Indeed, it was shown that VeA of A. niger migrates to the nucleus in the dark. Nuclear migration of VeA of A. nidulans depends on the interaction between its NLS and the $\alpha$ importin KapA (Stinnett et al. 2007). The A. niger genome contains a homoloque of this protein, suggesting that VeA of $A$. niger 

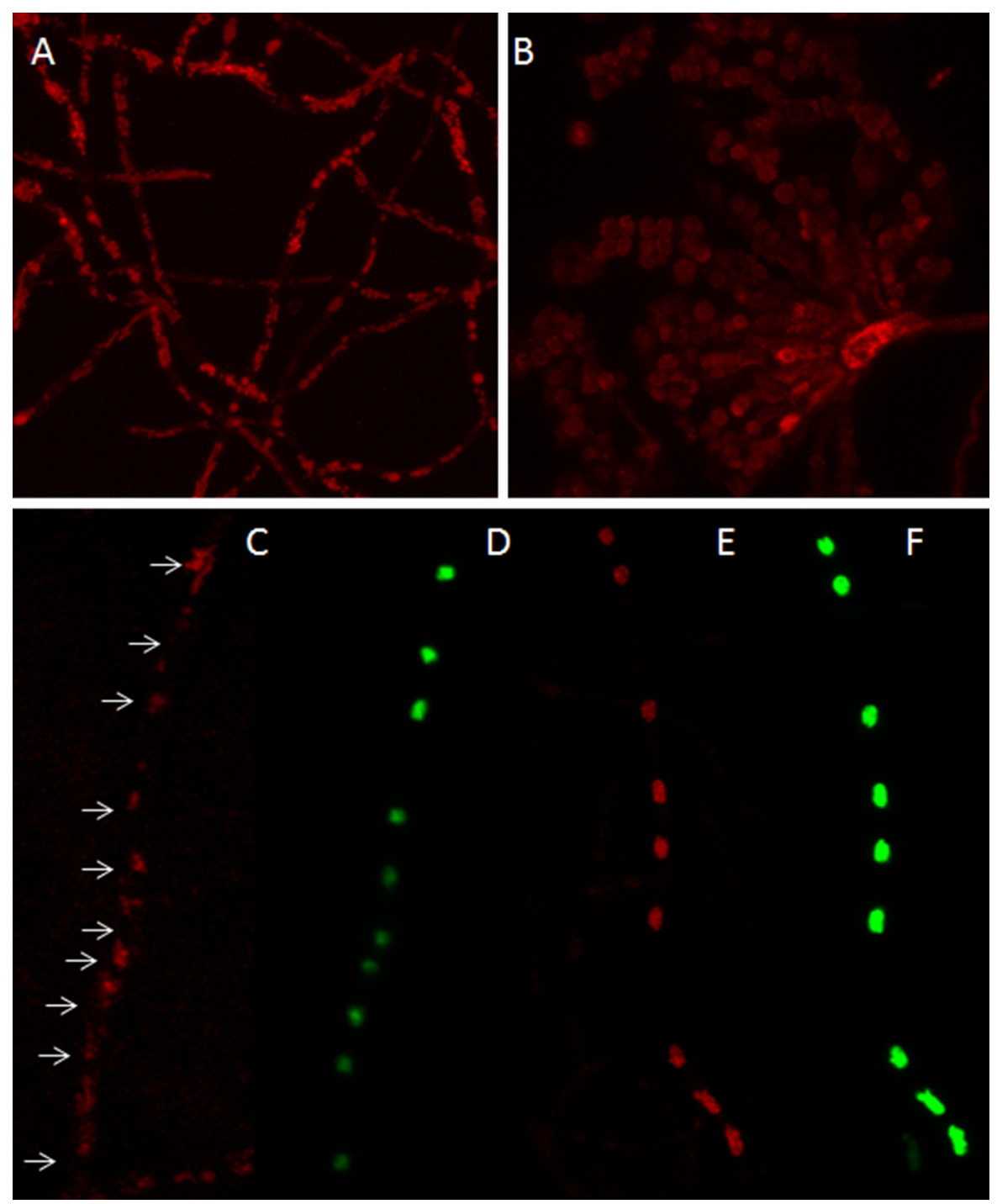

Fig. 6 Confocal fluorescence microscopy shows the presence of VeA-RFP both in the vegetative mycelium (a) and in conidiophores (b) of cultures that had been grown in the light $(1,200$ lux $)$. Localization of VeA in light $(\mathbf{c}, \mathbf{d})$ and dark $(\mathbf{e}, \mathbf{f})$ in

also co-migrates with KapA to the nucleus. Notably, the A. niger control strains and the $\Delta v e A$ strain did not show light dependent conidia formation as was shown in A. nidulans and A. parasiticus (Calvo et al. 2004; Bayram et al. 2010). Conidia and sclerotia formation seem also to be independent of light in Aspergillus carbonarius (Crespo-Sempere et al. 2013) and $A$. flavus (Duran et al. 2007), respectively.

Spatial positioning of conidiophores was affected in the $\Delta v e A$ colony. Sandwiched $\Delta v e A$ colonies mainly formed conidiophores at the sub-peripheral vegetative mycelium by localizing fluorescence of $\operatorname{VeA}-\mathrm{RFP}(\mathbf{c}$, e) and the nuclear targeted H2B-GFP fusion protein (d, f). Arrows indicate positions of nuclei

zone of the mycelium when conidiogenesis was induced by removing the upper PC membrane. In contrast, the control and the complemented strain formed conidiophores both in the center and at the subperiphery. Conidiophores of $\Delta v e A$ had shorter and thinner stalks and a reduced vesicle diameter. This phenotype cannot be explained by a reduced turgor pressure in the vegetative and aerial hyphae since total compatible solute levels were similar in the control strain and $\Delta v e A$. It may be that the phenotype is due to reduced levels of individual compatible solutes. 
Table 2 Accumulation (nmol solute $\mathrm{mg}^{-1}$ dry weight) of polyols, glucose and trehalose in vegetative mycelium and vegetative mycelium with conidia forming conidiophores of control strain RB\#210.1, $\Delta v e A$, and the complemented $\Delta v e A$ strain VC9

\begin{tabular}{|c|c|c|c|c|c|c|}
\hline & RB\#210.1myc & $\mathrm{RB} \# 210.1 \mathrm{myc}+\mathrm{con}$ & $\Delta v e A$ myc & $\Delta v e A$ myc + con & VC9 myc & VC9 myc + con \\
\hline Trehalose & $171 \pm 41$ & $59 \pm 16$ & $* 14 \pm 2$ & $* 16 \pm 0$ & $129 \pm 31$ & $84 \pm 6$ \\
\hline Glucose & $1 \pm 1$ & 0 & 0 & 0 & $5 \pm 5$ & 0 \\
\hline Erythritol & $23 \pm 5$ & $21 \pm 14$ & $* 73 \pm 3$ & $* 92 \pm 7$ & $* 93 \pm 19$ & $23 \pm 6$ \\
\hline Glycerol & 0 & $11 \pm 11$ & 0 & 0 & 0 & $26 \pm 21$ \\
\hline Mannitol & $332 \pm 27$ & $471 \pm 159$ & $341 \pm 15$ & $453 \pm 31$ & $416 \pm 99$ & $411 \pm 37$ \\
\hline Arabitol & 0 & 0 & 0 & 0 & 0 & 0 \\
\hline Total & $526 \pm 73$ & $562 \pm 201$ & $428 \pm 17$ & $561 \pm 38$ & $642 \pm 142$ & $543 \pm 27$ \\
\hline
\end{tabular}

* Indicates significant difference in sugar or polyol concentration when compared to wild-type (ANOVA $p<0.05$ )

Table 3 Accumulation (fmol per spore) of polyols, glucose and trehalose in conidia.of RB\#210.1, $\Delta v e A$, and the complemented $\Delta v e A$ strain (VC9)

\begin{tabular}{llll}
\hline & RB\#210.1 conidia & $\Delta v e A$ conidia & VC9 conidia \\
\hline Trehalose & $0.2 \pm 0$ & $* 0$ & $0.2 \pm 0.2$ \\
Glucose & 0 & 0 & $0.1 \pm 0.1$ \\
Erythritol & 0 & 0 & 0 \\
Glycerol & $3.8 \pm 0.5$ & $6.9 \pm 3.5$ & $4.9 \pm 4.7$ \\
Mannitol & $16.2 \pm 0.2$ & $* 9.0 \pm 3.7$ & $16.5 \pm 15.8$ \\
Arabitol & 0 & 0 & 0 \\
Total & $20.2 \pm 0.8$ & $15.9 \pm 7.2$ & $21.7 \pm 20.7$ \\
\hline
\end{tabular}

* Indicates significant difference in sugar or polyol concentration when compared to wild-type and VC9 (ANOVA $p<0.05$ )

Trehalose was absent in conidia of $\Delta v e A$ and it was 4-12 fold reduced in mycelium and mycelium with conidiophores and conidia. A. niger has three trehalose-6-phosphate synthase genes, tpsA-C, and three trehalose phosphate phosphatase genes, tppA-C (Svanstrom et al. 2014). In contrast to the other three deletion strains, $\triangle t p s A, \triangle t p p A$ and $\triangle t p p B$ strains showed lower trehalose levels. The only mutant that showed a morphological phenotype was $\Delta t p p A$. This strain produced 600 times less conidia per surface area and vesicle swelling of the conidiophore was virtually absent. These data also point to a role of trehalose in conidiophore formation.

The $\Delta v e A$ strain produced 17- (in the light) and 8- (in the dark) fold less spores when compared to the control strain. This difference can be explained by the morphology of the conidiophores. A twofold reduction of the vesicle diameter reduces its surface area fourfold, thus restricting sterigmata to develop. The length of the spore chains was also reduced evidenced from scanning electron microscopy and the threefold reduced diameter of mature $\Delta v e A$ conidiophores. Taking into account the reduced vesicle diameter and assuming similar sized sterigmata, it can be calculated that the wild-type and $\Delta v e A$ strains produce spore chains consisting of about 20 and 4 conidia, respectively. A fourfold reduction in vesicle surface and a fivefold reduced spore chain length would thus create a 20 -fold reduction in spore numbers, which agree with the numbers that were found by spore counting.

The conidia of $\Delta v e A$ were as viable as those of the wild-type. In fact, they germinated even faster when compared to the control strain. Yet, hyphal growth of the $\Delta v e A$ strain was affected. This was evidenced from a reduced radial growth and twofold reduced biomass formation. A similar role of VeA in biomass formation has been shown in A. parasiticus (Calvo et al. 2004). Reduced radial growth and biomass formation in A. niger was accompanied by vegetative hyphae that were growing wavy in an irregular way. Zig-zag or meandering hyphal growth has been observed in strains that lack microtubule-associated kinesin motor proteins or the teaA or teaR cell end markers that localize actin nucleation to sterol-rich apical membranes (Konzack et al. 2005). These data suggest that VeA of $A$. niger somehow functions in polar growth. Together, it can be concluded that VeA of $A$. niger has a strong impact of the abundance of this fungus in nature by stimulating growth and spore formation.

\section{References}

Bayram O, Braus GH (2011) Coordination of secondary metabolism and development in fungi: the velvet family of regulatory proteins. FEMS Microbiol Rev 36:1-24 
Bayram O, Braus GH, Fischer R, Rodriguez-Romero J (2010) Spotlight on Aspergillus nidulans photosensory systems. Fungal Genet Biol 47:900-908

Bayram O, Krappmann S, Ni M, Bok JW, Helmstaedt K, Valerius O, Braus-Stromeyer S, Kwon NJ, Keller NP, Yu JH, Braus GH (2008a) VelB/VeA/LaeA complex coordinates light signal with fungal development and secondary metabolism. Science 320:1504-1506

Bayram O, Krappmann S, Seiler S, Vogt N, Braus GH (2008b) Neurospora crassa ve-1 affects asexual conidiation. Fungal Genet Biol 45:127-138

Bennett JW (2010) An overview of the genus Aspergillus. In: Machida M, Gomi K (eds) Aspergillus: molecular biology and genomics. Caiser Academic Press, Portland, pp 1-17

Bleichrodt R (2012) Intercompartmental streaming in Aspergillus. PhD Thesis, University of Utrecht

Bok JW, Keller NP (2004) LaeA, a regulator of secondary metabolism in Aspergillus spp. Eukaryot Cell 3:527-535

Calvo AM, Bok J, Brooks W, Keller NP (2004) veA is required for toxin and sclerotial production in Aspergillus parasiticus. Appl Environ Microbiol 70:4733-4739

Crespo-Sempere A, Marin S, Sanchis V, Ramos AJ (2013) VeA and LaeA transcriptional factors regulate ochratoxin A biosynthesis in Aspergillus carbonarius. Int $\mathrm{J}$ Food Microbiol 166:479-486

de Bekker C, Wiebenga A, Aguilar G, Wösten HAB (2009) An enzyme cocktail for efficient protoplast formation in Aspergillus niger. J Microbiol Methods 76:305-306

de Bekker C, Bruning O, Jonker MJ, Breit TM, Wösten HAB (2011) Single cell transcriptomics of neighboring hyphae of Aspergillus niger. Genome Biol 12:R71

Duran RM, Cary JW, Calvo AM (2007) Production of cyclopiazonic acid, aflatrem, and aflatoxin by Aspergillus flavus is regulated by $v e A$, a gene necessary for sclerotial formation. Appl Microbiol Biotechnol 73:1158-1168

Geiser DM (2009) Sexual structures in Aspergillus: morphology, importance and genomics. Med Mycol 47:S21-S26

Horton P, Nakai K (1997) Better prediction of protein cellular localization sites with the $\mathrm{k}$ nearest neighbors classifier. Intern Conf Intell Syst Mol Biol 5:147-152

Kim H, Han K, Kim K, Han D, Jahng K, Chae K (2002) The veA gene activates sexual development in Aspergillus nidulans. Fungal Genet Biol 37:72-80

Konzack S, Rischitor PE, Enke C, Fischer R (2005) The role of the kinesin motor KipA in microtubule organization and polarized growth of Aspergillus nidulans. Mol Biol Cell 16:497-506

Krappmann S, Bayram O, Braus GH (2005) Deletion and allelic exchange of the Aspergillus fumigatus veA locus via a novel recyclable marker module. Eukaryot Cell 4:1298-1307

Krijgsheld P, Bleichrodt R, van Veluw GJ, Wang F, Muller WH, Dijksterhuis J, Wösten HAB (2013) Development in Aspergillus. Stud Mycol 74:1-29

Lagopodi AL, Ram AFJ, Lamers GE, Punt PJ, van den Hondel CAMJJ, Lugtenberg BJ, Bloemberg GV (2002) Novel aspects of tomato root colonization and infection by Fusarium oxysporum f. sp. radicis-lycopersici revealed by confocal laser scanning microscopic analysis using the green fluorescent protein as a marker. Mol Plant Microbe Interact 15:172-179
Li S, Myung K, Guse D, Donkin B, Proctor RH, Grayburn WS, Calvo AM (2006) FvVE1 regulates filamentous growth, the ratio of microconidia to macroconidia and cell wall formation in Fusarium verticillioides. Mol Microbiol 62:1418-1432

Merzlyak EM, Goedhart J, Shcherbo D, Bulina ME, Shcheglov AS, Fradkov AF, Gaintzeva A, Lukyanov KA, Lukyanov S, Gadella TW, Chudakov DM (2007) Bright monomeric red fluorescent protein with an extended fluorescence lifetime. Nat Methods 4:555-557

Myung K, Li S, Butchko RA, Busman M, Proctor RH, Abbas HK, Calvo AM (2009) FvVE1 regulates biosynthesis of the mycotoxins fumonisins and fusarins in Fusarium verticillioides. J Agric Food Chem 57:5089-5094

Ohm RA, de Jong JF, Berends E, Wang F, Wösten HAB, Lugones LG (2010) An efficient gene deletion procedure for the mushroom-forming basidiomycete Schizophyllum commune. World J Microbiol Biotechnol 26:1919-1923

Pel HJ, de Winde JH, Archer DB, Dyer PS, Hofmann G, Schaap PJ, Turner G, de Vries RP, Albang R, Albermann $\mathrm{K}$, Andersen MR, Bendtsen JD, Benen JA, van den Berg M, Breestraat S, Caddick MX, Contreras R, Cornell M, Coutinho PM, Danchin EG, Debets AJ, Dekker P, van Dijck PW, van Dijk A, Dijkhuizen L, Driessen AJ, d'Enfert C, Geysens S, Goosen C, Groot GS, de Groot PW, Guillemette T, Henrissat B, Herweijer M, van den Hombergh JP, van den Hondel CA, van der Heijden RT, van der Kaaij RM, Klis FM, Kools HJ, Kubicek CP, van Kuyk PA, Lauber J, Lu X, van der Maarel MJ, Meulenberg R, Menke H, Mortimer MA, Nielsen J, Oliver SG, Olsthoorn M, Pal K, van Peij NN, Ram AF, Rinas U, Roubos JA, Sagt CM, Schmoll M, Sun J, Ussery D, Varga J, Vervecken W, van de Vondervoort PJ, Wedler H, Wösten HA, Zeng AP, van Ooyen AJ, Visser J, Stam $H$ (2007) Genome sequencing and analysis of the versatile cell factory Aspergillus niger CBS 513.88. Nat Biotechnol 25:221-231

Punt PJ, Oliver RP, Dingemanse MA, Pouwels PH, van den Hondel CAMJJ (1987) Transformation of Aspergillus based on the hygromycin B resistance marker from Escherichia coli. Gene 56:117-124

Purschwitz J, Muller S, Kastner C, Fischer R (2006) Seeing the rainbow: light sensing in fungi. Curr Opin Microbiol 9:566-571

Sarikaya Bayram O, Bayram O, Valerius O, Park HS, Irniger S, Gerke J, Ni M, Han KH, Yu JH, Braus GH (2010) LaeA control of velvet family regulatory proteins for lightdependent development and fungal cell-type specificity. PLoS Genet 6:e1001226

Stinnett SM, Espeso EA, Cobeno L, Araujo-Bazan L, Calvo AM (2007) Aspergillus nidulans VeA subcellular localization is dependent on the importin alpha carrier and on light. Mol Microbiol 63:242-255

Svanstrom A, van Leeuwen MR, Dijksterhuis J, Melin P (2014) Trehalose synthesis in Aspergillus niger: characterization of six homologous genes, all with conserved orthologs in related species. BMC Microbiol 14:90

Vishniac W, Santer M (1957) The thiobacilli. Bacteriol Rev 21:195-213 
Wösten HAB, Moukha SM, Sietsma JH, Wessels JGH (1991) Localization of growth and secretion of proteins in Aspergillus niger. J Gen Microbiol 137:2017-2023

Wyatt TT, van Leeuwen MR, Wösten HAB, Dijksterhuis J (2014) Mannitol is essential for the development of stress resistant ascospores of Neosartorya fischeri. Fungal Genet Biol 64:11-24
Yang L, Ukil L, Osmani A, Nahm F, Davies J, De Souza CP, Dou X, Perez-Balaguer A, Osmani SA (2004) Rapid production of gene replacement constructs and generation of a green fluorescent protein-tagged centromeric marker in Aspergillus nidulans. Eukaryot Cell 3:1359-1362 